\title{
Method for Evaluation of Concentration of Solvent Molecules in a Specified Region of Regenerated Cellulose Solid
}

\author{
Sei-ichi Manabe* and Rumiko FujIOKA*,** \\ * Faculty of Human Environmental Science, Fukuoka Women's University, \\ 1-1-1 Kasumigaoka, Higashi-ku, Fukuoka 813-8529, Japan \\ ** Faculty of Engineering, Miyazaki University, 1-1 Gakuenkibana-nishi, Miyazaki 889-2155, Japan
}

(Received August 21, 1997)

\begin{abstract}
We propose a novel method for evaluation of the volume fraction, $v$, of solvent molecules in a specified region of a cellulose solid through dynamic viscoelasticity under the coexistence of solvents. The well-characterized two cuprammonium regenerated cellulose fibers were used. They were composed of primary particles with $c a$. $15 \mathrm{~nm}$ in size. A series of $n$-alcohols, poly(ethylene glycol)s, and poly(propylene glycol)s and glycerin were used as a hydrophilic molecules with OH groups. The dynamic storage modulus gave information on the state of the region where molecules could diffuse and peak temperature, $T_{\mathrm{fc}}$, of the mechanical loss tangent gave $v$ by putting $T_{\mathrm{fc}}$ into the semi-empirical equation. The results were as follows: (1) $T_{\mathrm{fc}}$ of $\beta_{\mathrm{a}}$ absorption due to the local twisting motion of a main chain shifted to lower temperature by water and/or methanol and to higher temperature for other solvents. (2) $v$ in the region related to the $\alpha_{\mathrm{sh}}$ absorption, originating from the segmental micro-brownian motion of the main chains located at the interface of the primary particle, showed a constant value for $n$-alcohols having less than eight carbon atoms per molecule. (3) $v$ in the region related to $\alpha_{\mathrm{a}}$ absorption, originating from the segmental micro-brownian motion of the main chain in the primary particle, increased when molecular weight per one $\mathrm{OH}$ group for the solvent decreased and/or the peak temperature of $\alpha_{\mathrm{a}}$ was low.
\end{abstract}

KEY WORDS Mechanical Absorption / Regenerated Cellulose / Free Volume / Diffusion / $\alpha_{\mathrm{a}}$ Absorption / $\beta_{\mathrm{a}}$ Absorption / $n$-Alcohol / Poly(ethylene glycol) / Poly(propylene glycol) / Glycerin /

Spinning technology is an established technology. At a solidification step in the technology, rigorous control of the environment of the manufacturing gives few faults in the higher order structures of a fiber. Although we can design and control higher order structures related to size more than $\mu \mathrm{m}$ using this technology, higher order structures having nanometer scale in size are not designed. To prepare the polymer solid with welldesigned higher order structure ranging from sub- $\mu \mathrm{m}$ to a whole body, novel technology is needed.

In finishing, diffusion of a solvent molecule into the polymer solid is an important factor which governs the higher order structure. Diffusion effects on the thermal molecular motion of the polymer chains in the solid result in the acceleration of the diffusion. Although there have been many reports on permselectivity ${ }^{1}$ and diffusion ${ }^{2}$ of materials in a polymer solid and higher order structure, only a few studies ${ }^{3,4}$ report the effects of the dye molecules in a fiber on the thermal molecular motion. In the diffusional study a polymer solid has been assumed to be a uniform fine structure represented by crystalline and non-crystalline regions. ${ }^{5}$ The relationship between higher order structure and thermal molecular motion has been investigated for regenerated cellulose fiber by us. ${ }^{6,7}$ Figure 1 summarizes the results. There are eight mechanical absorptions, $\alpha_{1}, \alpha_{21}, \alpha_{22}, \alpha_{\mathrm{sh}}, \alpha_{\mathrm{H}_{2} \mathrm{O}}, \alpha_{\mathrm{m}}$, $\beta_{\mathrm{a} 1}$, and $\beta_{\mathrm{a} 2}$ from higher temperature in the range between 600 and $150 \mathrm{~K}$. Four absorptions $\alpha_{1}, \alpha_{21}, \alpha_{22}$, and $\alpha_{\mathrm{sh}}$ originate from segmental micro-brownian motion and temperature locations are reflected in the density of the intermolecular hydrogen bonds. ${ }^{6} \alpha_{\mathrm{sh}}$ is related to the interfacial region of the primary particle. ${ }^{7} \alpha_{\mathrm{H}_{2} \mathrm{O}}$ may originate from the cooperative motion of absorbed $\mathrm{H}_{2} \mathrm{O}$ and polymer segment. ${ }^{6} \beta_{\mathrm{a} 1}$ and $\beta_{\mathrm{a} 2}$ absorptions originate from local twisting motion of the main chain in the amorphous region and $\beta_{\mathrm{a} 1}$ may be related to the interfacial region of the primary particle. ${ }^{7} \alpha_{\mathrm{m}}$ absorption is due to the melting of $\mathrm{H}_{2} \mathrm{O}$ adsorbed within the fine space among neighboring primary particles. ${ }^{7}$ The lower part of Figure 1 shows locations of regions that give absorption in primary particles with $15 \mathrm{~nm}$ in diameter. The straight lines shown by e.c.m. stand for extended chain molecules.

This study proposes a novel evaluation method for the concentration of a solvent in a given region and makes clear the effects of the solvent diffused into the solid on the thermal motion of a polymer chain. This method is applied to cuprammonium regenerated cellulose fibers whose higher order structure is well-characterized.

\section{THEORETICAL BACKGROUND}

The geometrical size of thermal molecular motion of a polymer solid has been evaluated from experimental data related to mechanical absorption of polymer chains. ${ }^{2,7,8}$ The size of a segment that gives the $\alpha_{a}$ absorption due to the micro-brownian motion of a polymer chain segment is between 2 and $10 \mathrm{~nm}$ in length or is 300 and 1500 in molecular weight. ${ }^{7,9}$ As for $\beta_{\mathrm{a}}$ absorption due to local twisting motion of a polymer chain, the moving unit is from 0.4 to $2 \mathrm{~nm}$ in length or molecular weight, from 60 to 300 . We assume the effects of the solvent diffused into a polymer solid on the thermal molecular motion are governed by (1) dispersion state of solvent molecules in the polymer solid and (2) molecular size of solvent compared with that of the moving unit.

The region where the solvent diffuses is referred to as the penetrable region. This region is related to the higher order structure, chemical affinity between solvent and cellulose, thermal molecular motion and solvent size. Thermal molecular motion of a cellulose chain is in- 

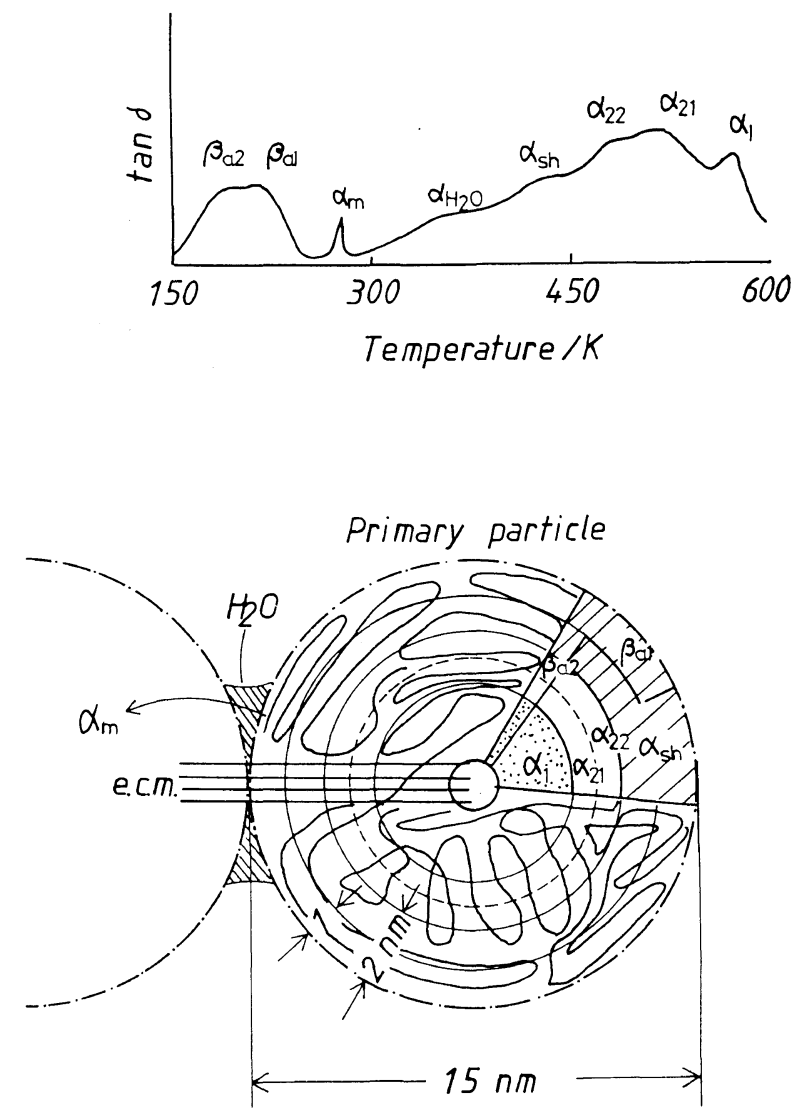

Figure 1. Schematic representation of regions related to dynamic absorption for regenerated cellulose: Upper side, temperature dependence of dynamic loss tangent $\tan \delta$. Symbol $\alpha$ stands for the micro-brounian motion in an amorphous region (these are named generically as $\alpha_{\mathrm{a}}$ absorption) and $\beta$ stands for local twisting motion of a main chain in an amorphous region (generically as $\beta_{\mathrm{a}}$ absorption). Lower side, location of the regions related to dynamic absorptions. Regenerated cellulose solid is composed of aggregates of primary particles (shown by a circle drown with chain line) having $15 \mathrm{~nm}$ in size. Symbol e.c.m. stands for the extended chain molecular chain in the primary particle. Regions related to $\beta_{\mathrm{a} 1}$ and $\alpha_{\mathrm{sh}}$ are distributed at interfacial zones of primary particles with $1 \mathrm{~nm}$ and $2 \mathrm{~nm}$ in thickness, respectively.

fluenced strongly by the higher order structure, and thus we may evaluate the penetrable region of the solvent related to the higher order structure from change in the thermal molecular motion by solvent. Mechanical loss $\operatorname{tangent} \tan \delta$ and storage modulus $E^{\prime}$ are representatives of thermal molecular motion.

\section{Change in Temperature Location of tan $\delta$ with Solvent}

The dispersion of a solvent in a cellulose solid is either (A), (B), or (C) shown in Figure 2 from the standpoint of effects on $\tan \delta$. Dispersion is expected to be explained on the basis of the diffusional behavior of the solvent into the regenerated cellulose solid and affinity among the molecules. Here, the cellulose solid is composed of primary particles. ${ }^{7}$

(A) Molecularly isolated state of solvent

A solvent molecule exists in a solid as an isolated state and the distance between solvent molecules is much more than the mean free path length of molecules in a gas. In this case, the free volume theory is not applicable for solvent molecules since the free volume of the molecule cannot be given by the free volume theory derived for a liquid state.

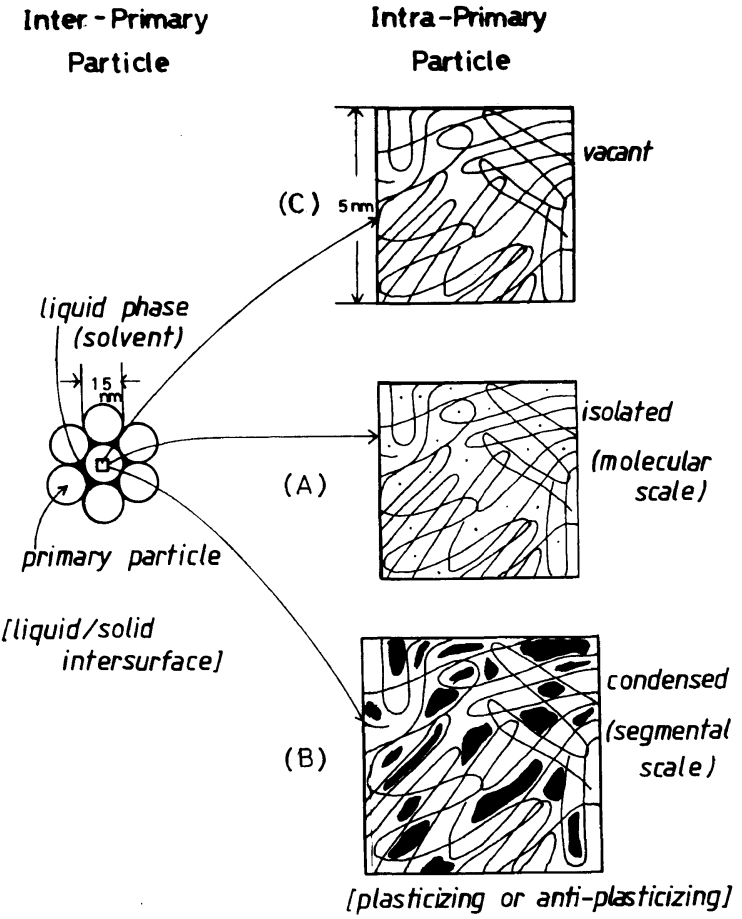

Figure 2. Three dispersion states of solvent in a polymer solid: Left side, solvent (shown as liquid phase) in the space of inter-primary particles. Right side, solvent molecules in a primary particle. Square frame indicates a sectional view in the primary particle. Length of a side is $5 \mathrm{~nm}$. String stands for a polymer chain. Filled zone stands for the liquid phase of the solvent. Dispersion state (A), solvent molecules are dispersed in isolated manner because of very small population; Dispersion state (B), solvent molecules dissolve in a primary particle as a liquid phase, that is, molecularly mixed state with polymer segments; Dispersion state (C), solvent molecules are not present in a primary particle but only in interfacial spaces of the particles.

(B) Dissolved state of solvents as liquid with cellulose

This state indicates that when we divide the region of solvents with size comparable to that of the cellulose segment related to the $\alpha_{\mathrm{a}}$ absorption, we find the solvent in every division. The solvent molecules within the division interact with each other. The solvent is considered to be a viscous fluid and the free volume theory may be applicable to express the contribution of the solvent to thermal molecularmotion of the chain.

(C) Dispersed state of solvent as the isolated liquid phase in cellulose solid

The size of the liquid phase is more than that of the segment. Interaction between a solvent and a cellulose chain is limited at the interface between two phases.

The difference in size between the segment and solvent molecule is another factor that affects absorption. The difference is classified as follows:

(1) Size of the solvent molecule is larger than that of the segment of $\alpha_{\mathrm{a}}$ absorption.

(2) Size of the solvent molecule ranges between the segment and the moving unit of $\beta_{\mathrm{a}}$ absorption.

(3) Size of the solvent molecule is less than that of the moving unit of $\beta_{\mathrm{a}}$ absorption.

(1), (2), and (3) for each dispersed state of (A), (B), or $(\mathrm{C})$ are realistic. For $(\mathrm{C})$, the free volume of the polymer solid other than the interface is not influenced by the solvent. The free volume theory, applicable only to the description of the liquid state, may not be applicable to the solvent for (A) due to isolated distribution 
like the diluted gas phase of solvents. Then, only in (1), (2), and (3) for (B), the free volume theory is useful for interpretation of the change in the temperature location of the mechanical absorption of the component polymer containing solvents. We may evaluate the concentration of the solvent in the region where the polymer is activated by the diffused solvent.

We assume that the free volume fraction of the solvent molecule is always larger than that of the polymer solid. $\alpha_{\mathbf{a}}$ absorption shifts to the lower temperature for both (B)-(1) and (B)-(2). $\beta_{\mathrm{a}}$ absorption shifts to the higher temperature side or disappears by the solvent. That is, anti-plasticizing effect ${ }^{7}$ occurs due to size effect. For (C)-(1) and (C)- (2), anti-plasticizing effect for $\beta_{\mathrm{a}}$ absorption appears in the vicinity of interfacial region of primary particles by the solvent. ${ }^{7}$ For (B)-(3), plasticizing effect by the solvent for $\alpha_{\mathrm{a}}$ and $\beta_{\mathrm{a}}$ is realized. When we know changes in the temperature of the mechanical absorption by the solvent molecules, we can evaluate the concentration of the solvent in the region related to the absorption through the free volume theory.

The shift of the absorption to the lower temperature is explained by the free volume theory as follows ${ }^{10}$ : The moving unit has inherent free volume fraction at a given temperature. After dissolving a solvent, sharing of the whole free volume supplied by the moving unit and solvent occurs and the free volume belonging to the solvent is utilized to activate the thermal motion of the moving unit.

We define the freezing temperature of the thermal motion causing a given dynamic absorption (for example, $\alpha_{\mathrm{a}}$ absorption) as $T_{\mathrm{f}}$, free volume fraction at the freezing temperature as $f_{\mathrm{f}}$, and thermal expansion coefficient of the free volume as $\alpha_{\mathbf{f}}$. The free volume fraction $f$ at temperature $T$ is given by,

$$
f=f_{\mathrm{f}}+\alpha_{\mathrm{f}}\left(T-T_{\mathrm{f}}\right) \quad T \geqq \mathrm{~T}_{\mathrm{f}}
$$

The free volume fraction $f(s)$ for a solvent molecule in a liquid state is assumed to be,

$$
f(s)=f_{\mathrm{f}}(s)+\alpha_{\mathrm{f}}(s) \quad\left(T-T_{\mathrm{f}}(s)\right)
$$

where $f_{\mathrm{f}}(s)$ is the free volume fraction frozen in the glassy state, $T_{\mathrm{f}}(s)$ the glass transition temperature, and $\alpha_{\mathrm{f}}(s)$ the thermal expansion coefficient of the free volume fraction for the solvent. The volume fraction of the solvent in a given region is represented by $v$ and the free volume fraction $f_{\mathrm{c}}$ for the region with the solvent is derived on the assumption of the additivity of the free volume and is given by,

$$
f_{\mathrm{c}}=(1-v) f+v f(s)
$$

Even when the solvent is present, the free volume fraction to be frozen is assumed to be constant $f_{\mathrm{f}}$ at peak temperature of $\alpha_{\mathrm{a}}$ absorption $T_{\mathrm{fc}}$. By substitution of the relations of $f_{\mathrm{c}}=f_{\mathrm{f}}$ at $T=T_{\mathrm{fc}}$, eq 1 and eq 2 into eq 3 , eq 4 is derived,

$$
\frac{v}{1-v}=\frac{T_{\mathrm{f}}-T_{\mathrm{fc}}}{T_{\mathrm{fc}}-T_{\mathrm{f}}(s)}\left(\frac{\alpha_{\mathrm{f}}}{\alpha_{\mathrm{f}}(s)}\right)
$$

$\alpha_{\mathrm{f}}, \alpha_{\mathrm{f}}(s), T_{\mathrm{f}}$ and $T_{\mathrm{f}}(s)$ are inherent values of cellulose and solvent, and are determined when the polymer and solvents are given. Consequently, when we observe $T_{\mathrm{fc}}$, we can evaluate $v$ by putting $T_{\mathrm{fc}}$ into eq 4 .
The experimental values of $\alpha_{\mathrm{f}} / \alpha_{\mathrm{f}}(s)$ have not been determined and may be distributed between 0.45 (for example; methanol) and 3.0 (water) for organic solvents. The ratio of $T_{\mathrm{f}}(s)$ to the melting point of the solvent $T_{\mathrm{m}}(s)$ is between 0.5 of water ${ }^{11,12}$ and 0.6 of toluene. ${ }^{13}$ With 0.5 as the values of $\alpha_{\mathrm{f}} / \alpha_{\mathrm{f}}(s)$ and $T_{\mathrm{f}}(s) / T_{\mathrm{m}}(s)$ in eq 4 , we get eq 5 as approximate equation.

$$
v=\frac{\left(1-T_{\mathrm{fc}} / T_{\mathrm{f}}\right)}{\left(1+\left(T_{\mathrm{fc}}-T_{\mathrm{m}}\right) / T_{\mathrm{f}}\right)}
$$

when we use $2 / 3$ as for $\alpha_{\mathrm{f}} / \alpha_{\mathrm{f}}(s)$ and 0.5 for $T_{\mathrm{f}}(s) / T_{\mathrm{m}}(s)$, we get

$$
v=\frac{\left(1-T_{\mathrm{fc}} / T_{\mathrm{f}}\right)}{\left(1+0.5\left(T_{\mathrm{fc}} / T_{\mathrm{f}}\right)-0.75\left(T_{\mathrm{m}} / T_{\mathrm{f}}\right)\right)}
$$

Effects of Solvent Molecule on $E^{\prime}$

Even if the solvent content in a cellulose solid is constant, $E^{\prime}$ varies depending on the dispersion state of the solvent. $E^{\prime}$ at the temperature, where $\beta_{\mathrm{a}}$ absorption is frozen, is given by the theoretical equation derived by Uemura and Takayanagi. ${ }^{14}$

$E^{\prime}$ at temperatures above $\beta_{\mathrm{a}}$ absorption depends on the fraction of the molecular chains contributing to absorption and relaxation time for three dispersion states (A), (B), and (C). The effects of the solvent on $E^{\prime}$ can be explained through change in the thermal molecular motion by the solvent. Since change is described by the free volume theory for the dispersion state (B), change in $E^{\prime}$ by the solvent indicates the region where the solvent diffuses.

When we observe $E^{\prime}$ and calculate $E^{\prime} /(1-v)$ at a given temperature, we evaluate the penetrable region without knowing $T_{\mathrm{fc}}$ as discussed above. $E^{\prime}$ values before and after mechanical dispersion for each absorption are determined in advance from experimental $E^{\prime}$ at the observation temperature covering $\alpha_{\mathrm{a}}$ and $\beta_{\mathrm{a}}$ absorptions. When the observed $E^{\prime}$ with solvent coincides with the corresponding $E^{\prime}$ at the temperature higher than $\alpha_{\mathrm{a}}$ absorption, we conclude that the solvent diffuses into the region in which the polymer segments related to absorption exist. When the observed $E^{\prime}$ is corresponding to $E^{\prime}$ at the lower temperature than $\alpha_{\mathrm{a}}$ absorption, the solvent cannot diffuse into the region. When $E^{\prime}$ is the value in the temperature range of the $\alpha_{\mathrm{a}}$ absorption, we conclude that the solvent diffuses into a part of the region in question and/or the concentration of the molecule in the region is so small that $T_{\mathrm{fc}}$ is higher than the observation temperature of $E^{\prime}$.

The prerequisite for application of the evaluation method of the penetrable region from $E^{\prime}$ is that the measuring temperature should be lower than the immersing temperature of the solvent. Even in the case that the prerequisite is satisfied, there are some risks of miss-judgment when the measuring temperature is far lower than $T_{\mathrm{fc}}$. This drawback is solved by taking $T_{\mathrm{fc}}$ into consideration.

\section{EXPERIMENTAL}

\section{Sample Preparation}

Two cuprammonium regenerated cellulose fibers BMM40 and NP were used. The detailed description of 
Table I. Structural characteristics of BMM40 and NP

\begin{tabular}{lll}
\hline \multicolumn{1}{c}{ Sample code } & BMM40 & NP \\
\hline Outer diameter $/ \mu \mathrm{m}$ & 350 & 18 \\
X-Ray crystallinity $/ \%$ & $0-2$ & 54 \\
Chain orientation $\mathrm{Fb} /-$ & 0.0 & 0.45 \\
Birefringence at center $\Delta n_{\mathrm{c}}$ & 0.0016 & 0.0382 \\
Shape of aggregation unit & Particle & Micro-fibril \\
Size of the unit $/ \mathrm{nm}$ & 15 and 70 & 50 \\
\hline
\end{tabular}

the fine structure of BMM40 has been given in the previous report. ${ }^{7}$ Table I summarizes the structural characteristics of BMM40 and NP. After drying at $298 \mathrm{~K}$ the samples were immersed in a given solvent at $298 \mathrm{~K}$ for more than $48 \mathrm{~h}$ when no description was given. Glycerin and the series of $n$-alcohols, poly(ethylene glycol)s, and poly(propylene glycol)s were used as immersing liquids.

\section{Measurement}

Dynamic viscoelasticity in a solvent was measured by the method proposed by us ${ }^{7}$ previously using Rheovibron DDV-II manufactured by Toyo-Boldwin Co., Ltd., Japan. The sample was immersed in the solvent in the hollow space of the hollow fiber used as the enclosure in advance. Measuring frequency was $110 \mathrm{~Hz}$ and heating rate, $3 \mathrm{~K} \mathrm{~min}^{-1}$. The relative error of $E^{\prime}$ was less than $10 \%$ and that of $\tan \delta$, less than $3 \%$.

\section{RESULTS AND DISCUSSION}

Temperature Dependence of Dynamic Viscoelasticity in $n$-Alcohols for BMM40

The diffusion of a solvent may occur through as follows: (1) diffusion during preservation at $298 \mathrm{~K}$ in the solvent and then diffusion equilibrium is attained, and (2) accelerated diffusion by elevating temperature in measurement. Both diffusions may coexist. When (2) diffusion occurs, decrease in $E^{\prime}$ with increasing temperature must be more conspicuous than (1) diffusion because of the additional decrease due to plasticizing of the solvent. Figure 3 shows a comparison of samples preserved for 4 and 40 day at $298 \mathrm{~K}$ in glycerin. The sample of NP is composed of densely packed primary particles and diffusion of a solvent is more difficult than for BMM40. The dependence of $E^{\prime}$ on temperature for 4 day preservation shows abrupt decrease in the temperature range of $370 \mathrm{~K}$ and $400 \mathrm{~K}$ compared with dry air. In the case of 40 day preservation, $E^{\prime}$ below $370 \mathrm{~K}$ is smaller than that of 4 days resulting in gentle slope of $E^{\prime} v s$. temperature curve. Consequently, at $370 \mathrm{~K}$ and $400 \mathrm{~K}$, (2) diffusion is pronounced for the sample of 4 day preservation. We easily confirm the contribution of (2) diffusion to the solvent diffusion from the steep slope of $E^{\prime}$. Figure 4 shows the temperature dependence of $E^{\prime}$ and $\tan \delta$ for BMM40 in various $n$-alcohols. The range shown by arrows with the name of the dynamic absorption such as $\beta_{\mathrm{a}}$ and $\alpha_{\mathrm{sh}}$ indicates the range of $E^{\prime}$ that corresponds to absorptions such as $\beta_{\mathrm{a}}$ and $\alpha_{\mathrm{sh}}$, respectively. $E^{\prime}$ and $\tan \delta$ curves shift to the lower temperature side when the number of carbon atoms in a solvent decreases by rough estimation. More detailed examination indicated: (1) The temperature of $\beta_{\mathrm{a}}$ ab-

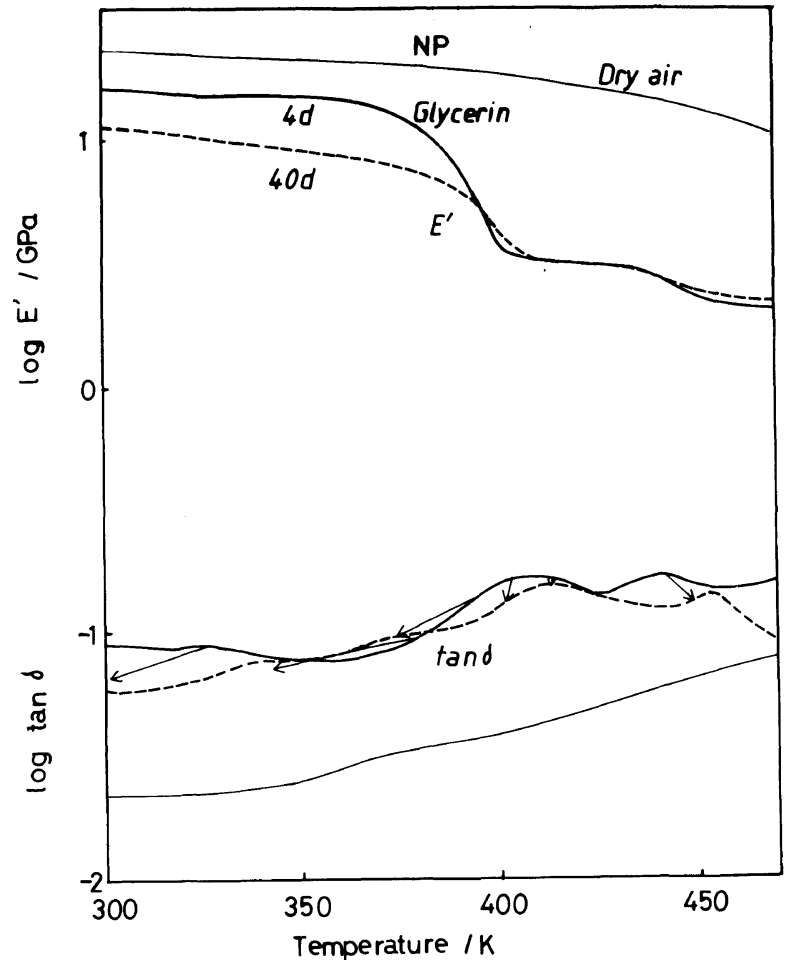

Figure 3. Temperature dependence of viscoelasticity of NP within glycerin: Preservation period is 4 days (full line) and 40 days (broken line). Arrows indicate directions of change due to the long preservation. Thin full line stands for data in dry air.

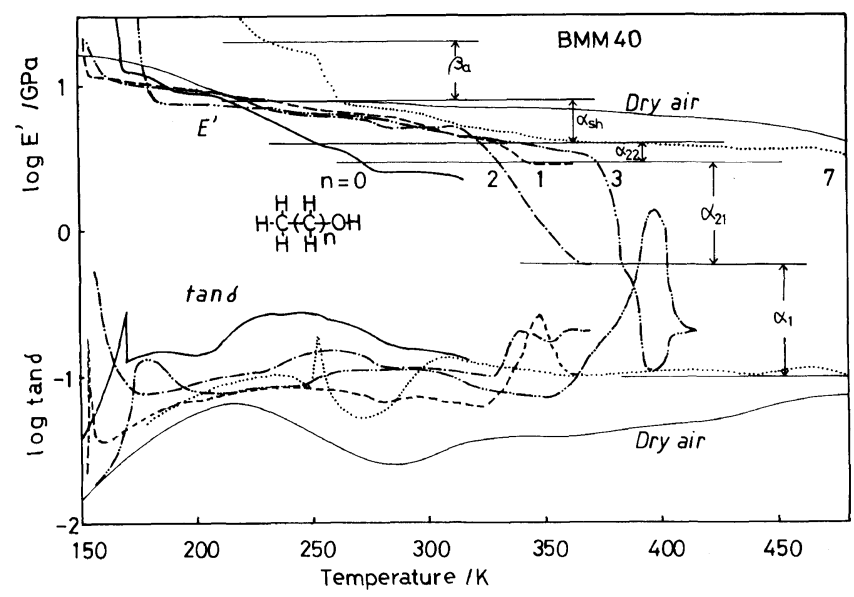

Figure 4. Temperature dependence of $E^{\prime}$ and $\tan \delta$ for BMM40 in various $n$-alcohols: $n$ indicates number of $\left(\mathrm{CH}_{2}\right)$ in the chemical formula of $\mathrm{CH}_{3}\left(\mathrm{CH}_{2}\right)_{n} \mathrm{OH}$ for $n$-alcohols. $E^{\prime}$ range shown by arrow indicates relaxation intensity originated by the dynamic absorption denoted by the symbols of $\alpha$ and $\beta$. Thin full line stands for data in dry air.

sorption shifts to the lower temperature side by methanol $(n=0)$ and to the higher temperature for $n \geqq 1$.

(2) By methanol, absorptions of $\alpha_{\text {sh }}$ and $\alpha_{22}$ shift to the lower temperature side although the shift is not clear for $\alpha_{21}$ and $\alpha_{1}$ absorptions.

(3) For $n=2$ (n-propanol) and 3 (n-butanol), $\alpha_{21}$ absorption shifts to the lower temperature and $\alpha_{1}$ absorption shifts also to the lower side for $n=3$. For (2) diffusion of these solvents into regions related to $\alpha_{21}$ and $\alpha_{1}$ is dominant. The $E^{\prime}$-temperature curve for $n=3$ shows a minimum at $c a .390 \mathrm{~K}$. Weight loss of the sample after increase in $E^{\prime}$ occurred. These data indicate $\mathrm{H}_{2} \mathrm{O}$ in a sample and/or the solvent may evaporate in this 


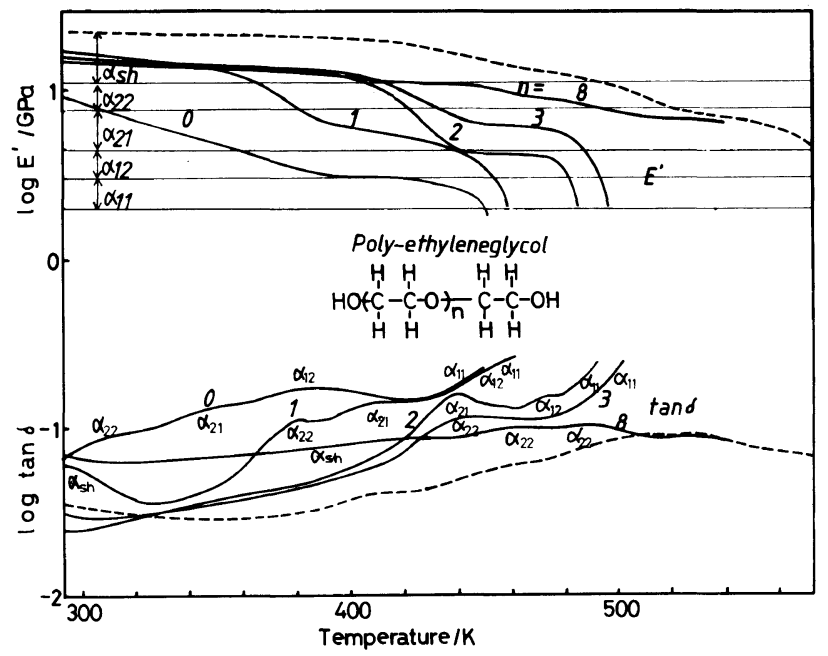

Figure 5. Temperature dependence of $E^{\prime}$ and $\tan \delta$ for NP in various poly(ethylene glycol) (PEG): $n$ indicates repeating number of $\left(\mathrm{C}_{2} \mathrm{H}_{4} \mathrm{O}\right)$ in the chemical formula of $\mathrm{HO}\left(\mathrm{C}_{2} \mathrm{H}_{4} \mathrm{O}\right)_{n} \mathrm{C}_{2} \mathrm{H}_{4} \mathrm{OH}$ of $\mathrm{PEG}$. $E^{\prime}$ range shown by arrow has same meaning as in Figure 4 . Broken line corresponds to data in dry air.

temperature range. (4) Even for $n=7$ ( $n$-octanol), $\alpha_{\mathrm{sh}}$ absorption shifts to the lower temperature side but $\alpha_{22}$, $\alpha_{21}$, and $\alpha_{\text {sh }}$ do not.

\section{Temperature Dependence of Dynamic Viscoelasticity in} Poly(ethylene glycol)s for NP

Figure 5 shows the temperature dependence of $E^{\prime}$ and $\tan \delta$ for NP in poly(ethylene glycol) (PEG) with various molecular weights. $n$ indicates the repeating number of $\left(\mathrm{C}_{2} \mathrm{H}_{4} \mathrm{O}\right)$ in $\mathrm{PEG}$ whose chemical structure is represented by $\mathrm{HO}\left(\mathrm{C}_{2} \mathrm{H}_{4} \mathrm{O}\right)_{n} \mathrm{C}_{2} \mathrm{H}_{4} \mathrm{OH}$. $\alpha_{1}$ absorption is separated conveniently into two such as $\alpha_{12}$ and $\alpha_{11}$ from the lower temperature side. Whether $\alpha_{1}$ absorption is composed of two absorptions or single is not clear but two absorptions were observed after the diffusion of ethylene glycol $(n=0)$. The names of various absorptions on the $\tan \delta$ curve were decided on from $E^{\prime}$. In general, when $n$ decreases all $\alpha_{\mathrm{a}}$ absorptions such as $\alpha_{\mathrm{sh}}, \alpha_{22}, \alpha_{21}$, and $\alpha_{1}$ absorptions shift to the lower temperature. More detailed examination of Figure 5 indicated: (1) Ethylene glycol diffuses into regions related to $\alpha_{\mathrm{sh}}, \alpha_{22}, \alpha_{21}$, and $\alpha_{1}$. Diffusion into the region related to $\alpha_{11}$ absorption is governed by (2) diffusion. (2) When $n$ increases, diffusion into the regions related to $\alpha_{22}, \alpha_{21}$, and $\alpha_{1}$ is mainly governed by (2) diffusion. (3) Even for $n=8$ (PEG with molecular weight of 600), the molecule easily diffuses into the region related to $\alpha_{\mathrm{sh}}$. The diffusion into the region related to $\alpha_{1}$ absorption may be governed by affinity between the cellulose, solvent, and molecular size. For $\alpha_{22}$ and $\alpha_{21}$ absorptions, affinity seems more dominant than size, and for $\alpha_{\text {sh }}$, only affinity may be dominant.

\section{Temperature Dependence of Dynamic Viscoelasticity in Poly(propylene glycol) for NP}

Figure 6 shows the temperature dependence of $E^{\prime}$ and $\tan \delta$ for NP in poly(propylene glycol) (PPG) with various molecular weights. For reference the results of PEG600 are included. $n$ indicates the repeating number of $\left(\mathrm{C}_{3} \mathrm{H}_{6} \mathrm{O}\right)$ in PPG whose chemical structure is $\mathrm{HO}\left(\mathrm{C}_{3} \mathrm{H}_{6} \mathrm{O}\right)_{n} \mathrm{C}_{3} \mathrm{H}_{6} \mathrm{OH}$. After the sample was cooled to $280 \mathrm{~K}$, temperature was increased. The dependence

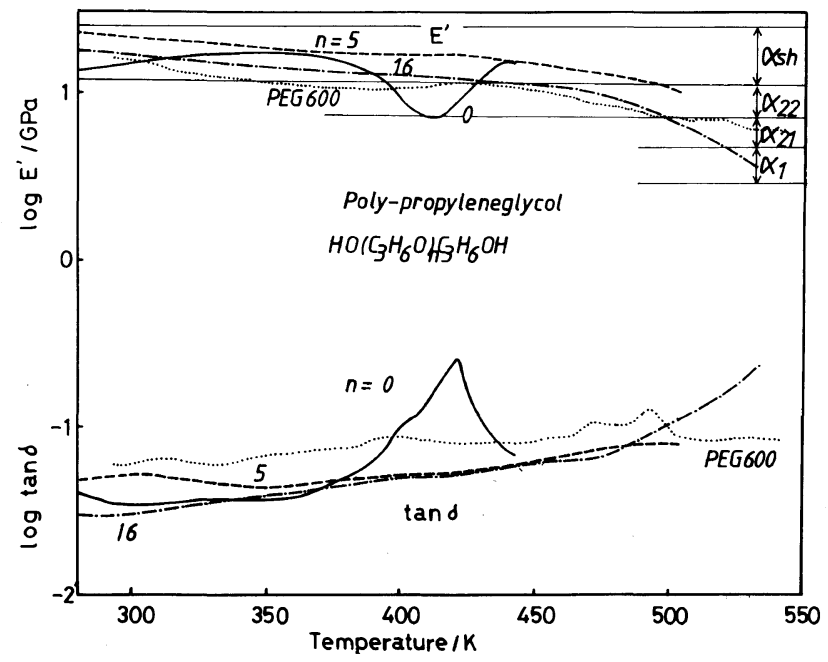

Figure 6. Temperature dependence of $E^{\prime}$ and $\tan \delta$ for NP in poly(propylene glycol) (PPG) with various molecular weights: $n$ indicates repeating number of $\left(\mathrm{C}_{3} \mathrm{H}_{6} \mathrm{O}\right)$ in $\mathrm{PPG}$ with the chemical formula of $\mathrm{HO}\left(\mathrm{C}_{3} \mathrm{H}_{6} \mathrm{O}\right)_{n} \mathrm{C}_{3} \mathrm{H}_{6} \mathrm{OH}$. Dotted lines correpond to data for PEG with molecular weight of 600 (PEG600 in short). Starting temperature of measurement was $280 \mathrm{~K}$. Arrows have same meaning as in Figure 4.

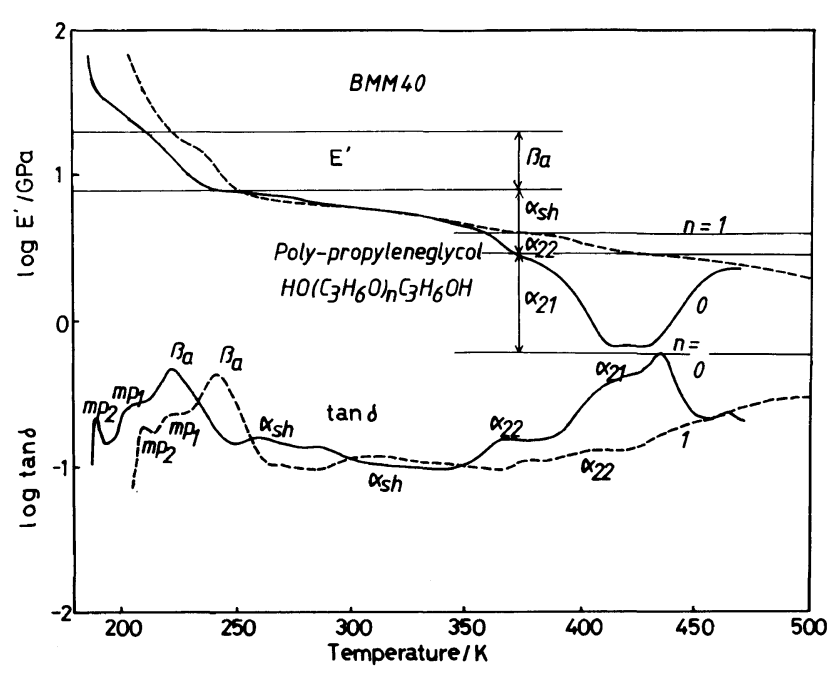

Figure 7. Temperature dependence of $E^{\prime}$ and $\tan \delta$ for BMM40 in PPG: Full line, propyleneglycol $(n=0)$; Broken line, dipropyleneglycol $(n=1)$. Starting temperature of measurement was $190 \mathrm{~K}$. Arrows have same meaning as in Figure 4.

of $E^{\prime}$ and $\tan \delta$ on $n$ is not simple. Apparently, when $n$ is five, diffusion into the region related to $\alpha_{\text {sh }}$ becomes difficult. For propylene glycol $(n=0)$, some solvent in the regions related to $\alpha_{\mathrm{sh}}$ is desorbed during heating to $370 \mathrm{~K}$ and $\alpha_{22}$ absorption occurs. After activation of $\alpha_{22}$ absorption, desorption of the solvent in the region related to $\alpha_{22}$ was accelerated. The occurrence of desorption means the concentration of the solvent after the adsorption equilibrium decreases with increase in temperature. The negative dependence of the adsorption on temperature indicates propylene glycol is adsorbed mainly through entalpy effect. Even for $n=16$, the PPG molecule penetrates the region related to $\alpha_{\text {sh }}$.

Figure 7 shows the temperature dependence of $E^{\prime}$ and $\tan \delta$ for $\mathrm{BMM} 40$ in propylene glycol $(n=0)$ and dipropylene glycol $(n=1)$. The sample was cooled to $190 \mathrm{~K}$ and temperature increased. Since the porosity of BMM40 is higher than that of NP and crystallinity is 
S. MANABE and R. FuJIOKA

Table II. Peak temperatures of $\tan \delta\left(T_{\mathrm{fc}}\right)$ for various solvents (in K)

\begin{tabular}{|c|c|c|c|c|c|c|}
\hline Solvent/Sample & $\beta_{\mathrm{a} 2}$ & $\beta_{\mathrm{a} 1}$ & $\alpha_{\mathrm{sh}}$ & $\alpha_{22}$ & $\alpha_{21}$ & $\alpha_{1}$ \\
\hline Dry air/BMM40 & 200 & 230 & 453 & 490 & 520 & 570 \\
\hline Air with $\mathrm{RH}=80 \% / \mathrm{BMM} 40$ & 185 & 212 & 263 & 333 & 360 & \\
\hline \multicolumn{7}{|l|}{$n$-Alcohols $\mathrm{CH}_{3}\left(\mathrm{CH}_{2}\right)_{n} \mathrm{OH}$} \\
\hline$n=0 / \mathrm{BMM} 40$ & 198 & 229 & 240 & 268 & 288 & \\
\hline 1/BMM40 & ca. 210 & ca. 260 & ca. 290 & ca. 340 & & \\
\hline 2/BMM40 & & $223^{\mathrm{a}}$ & 287 & 340 & 355 & \\
\hline 3/BMM40 & & $223^{a}$ & 273 & 370 & & \\
\hline 7/BMM40 & & $230^{\mathrm{a}}$ & 310 & 465 & & \\
\hline \multicolumn{7}{|l|}{ Poly(ethylene glycol)s } \\
\hline \multicolumn{7}{|l|}{$\mathrm{HO}\left(\mathrm{C}_{2} \mathrm{H}_{4} \mathrm{O}\right)_{n} \mathrm{C}_{2} \mathrm{H}_{4} \mathrm{OH}$} \\
\hline$n=0 / \mathrm{NP}$ & & & $<300$ & 308 & 350 & $383,440^{\mathrm{b}}$ \\
\hline $1 / \mathrm{NP}$ & & & 300 & 380 & 410 & 450 \\
\hline $2 / \mathrm{NP}$ & & & 360 & 435 & 440 & $475,485^{b}$ \\
\hline \multicolumn{7}{|l|}{ Poly(propylene glycol)s } \\
\hline \multicolumn{7}{|l|}{$\mathrm{HO}\left(\mathrm{C}_{3} \mathrm{H}_{6} \mathrm{O}\right)_{n} \mathrm{C}_{3} \mathrm{H}_{6} \mathrm{OH}$} \\
\hline$n=0 / \mathrm{NP}$ & & & $<300$ & 403 & \multicolumn{2}{|l|}{$>443$} \\
\hline $5 / \mathrm{NP}$ & & & 425 & $>473$ & & \\
\hline $16 / \mathrm{NP}$ & & & 373 & 503 & 513 & 533 \\
\hline 0/BMM40 & & $223^{a}$ & 261 & 368 & 413 & $>473$ \\
\hline 1/BMM40 & & $242^{\mathrm{a}}$ & 312 & 404 & 505 & $>533$ \\
\hline Glycerin/NP 4 day & & & 327 & 373 & 400 & $413,443^{b}$ \\
\hline 40 day & & & 300 & 350 & 400 & $413,447^{b}$ \\
\hline
\end{tabular}

${ }^{a}$ Not separated clearly. ${ }^{b}$ Separated into two absorptions.

Table III. Volume fraction $v$ of a solvent in a specified region

\begin{tabular}{|c|c|c|c|c|}
\hline Solvent/Sample & $\alpha_{\mathrm{sh}}$ & $\alpha_{22}$ & $\alpha_{21}$ & $\alpha_{1}$ \\
\hline Air with $\mathrm{RH}=80 \% / \mathrm{BMM} 40$ & 0.48 & 0.32 & 0.31 & $<0.30$ \\
\hline Water/BMM40 & $>0.31$ & $>0.44$ & $>0.48$ & $>0.51$ \\
\hline \multicolumn{5}{|l|}{$n$-Alcohols $\mathrm{CH}_{3}\left(\mathrm{CH}_{2}\right)_{n} \mathrm{OH}$} \\
\hline$n=0 / \mathrm{BMM} 40$ & 0.48 & 0.45 & 0.45 & $<0.40$ \\
\hline 1/BMM40 & ca. 0.35 & ca. 0.30 & $<0.32$ & $<0.38$ \\
\hline 3/BMM40 & 0.40 & 0.26 & $<0.20$ & $<0.20$ \\
\hline 7/BMM40 & 0.31 & 0.05 & $<0.03$ & $<0.03$ \\
\hline \multicolumn{5}{|l|}{ Poly(ethylene glycol)s } \\
\hline \multicolumn{5}{|l|}{$\mathrm{HO}\left(\mathrm{C}_{2} \mathrm{H}_{4} \mathrm{O}\right)_{n} \mathrm{C}_{2} \mathrm{H}_{4} \mathrm{OH}$} \\
\hline$n=0 / \mathrm{NP}$ & $>0.34$ & 0.37 & 0.33 & $0.33^{\mathrm{a}}$ \\
\hline $1 / \mathrm{NP}$ & 0.34 & 0.22 & 0.21 & 0.21 \\
\hline $2 / \mathrm{NP}$ & 0.21 & 0.11 & 0.15 & $0.17^{\mathrm{a}}$ \\
\hline $8 / \mathrm{NP}$ & 0.11 & 0.06 & 0.0 & 0.0 \\
\hline \multicolumn{5}{|l|}{ Poly(propylene glycol)s } \\
\hline \multicolumn{5}{|l|}{$\mathrm{HO}\left(\mathrm{C}_{3} \mathrm{H}_{6} \mathrm{O}\right)_{n} \mathrm{C}_{3} \mathrm{H}_{6} \mathrm{OH}$} \\
\hline$n=0 / \mathrm{NP}$ & $>0.32$ & 0.17 & $<0.14$ & $<0.14$ \\
\hline $5 / \mathrm{NP}$ & 0.02 & $<0.03$ & 0.0 & 0.0 \\
\hline $16 / \mathrm{NP}$ & 0.05 & 0.0 & 0.0 & 0.0 \\
\hline 0/BMM40 & 0.44 & 0.23 & 0.19 & $<0.15$ \\
\hline 1/BMM40 & 0.32 & 0.17 & 0.03 & $<0.05$ \\
\hline Glycerin/NP 4 day & 0.28 & 0.25 & 0.22 & $0.25^{\mathrm{a}}$ \\
\hline 40 day & 0.35 & 0.30 & 0.22 & $0.24^{\mathrm{a}}$ \\
\hline
\end{tabular}

${ }^{a}$ Mean value for two absorptions. 
lower, the diffusion of solvents is expected to be easier than for NP. $E^{\prime}$ in PPG with $n=0$ shows a minimum at the temperature range between 410 and $440 \mathrm{~K}$. The desorption of propylene glycol in this temperature range was evident. The solvent can penetrate the regions related to $\alpha_{\mathrm{sh}}, \alpha_{22}$, and $\alpha_{21}$ absorptions. Compared with NP, propylene glycol diffuses into BMM40 more easily. As for $\beta_{\mathrm{a}}$ absorption, the peak temperature elevates with the solvents. Two melting points of $m p_{1}$ and $m p_{2}$ are observed in both solvents of $n=0$ and $n=1 . \mathrm{mp}_{1}$ corresponds to the melting point of the bulk pure solvent and $\mathrm{mp}_{2}$ may be the melting point of the solvent adsorbed within fine spaces among neighboring primary particles. ${ }^{7}$

By the results in Figures 6 and 7, we can summerize the diffusional behavior of PPG into a cellulose solid as follows:

(1) $\beta_{\mathrm{a}}$ absorption shifts to higher temperature side with PPG. The shift increases with $n$.

(2) $\alpha_{\mathrm{sh}}, \alpha_{22}$, and $\alpha_{21}$ absorptions shift to the lower temperature side with PPG.

(3) Propylene glycol penetrates the regions related to $\alpha_{21}$ only at lower temperture than $280 \mathrm{~K}$.

(4) When $n$ increases to five, the diffusion into the region related to $\alpha_{\mathrm{sh}}$ becomes difficult and when $n$ increases over five to sixteen, diffusion is easy.

PPG may disturb the movement of $\beta_{\mathrm{a}}$ absorption, that is, anti-plasticizing may appear as shown in (1). For $\alpha_{\mathrm{a}}$ absorption, plasticizing of PPG appears as in (2). The results (3) and (4) indicate the diffusion rate of PPG possibly depends strongly on the molecular weight of PPG through the affinity and the molecular size. The increase in $n$ may decrease affinity due to decrease in density of $\mathrm{OH}$ groups in a molecule resulting in increase in diffusion rate as is shown for $n \geqq 5$. The increase in $n$ in the range of $n \leqq 4$ may decrease the diffusion rate.

\section{Solvent Concentration in a Given Amorphous Region Evaluated through Eq 6}

$T_{\mathrm{fc}}$ can be determined from $E^{\prime}$ and $\tan \delta$ given in Figures 3, 4, 5, 6, and 7. Table II summarizes $T_{\mathrm{fc}}$ of all absorptions for various solvents. At $n$ less than two, the melting point $T_{\mathrm{m}}$ of solvents other than $n$-alcohols was determined by the temperature dependence of the visco- elasticity. At $n$ more than three, the melting point was assumed same as that of $n=2$. These $T_{\mathrm{fc}}$ are substituted into eq 6 and we can evaluate $v$ for each amorphous region. Table III summarizes $v$ for regions related to the dynamic absorptions. The results are summarized as follows: (1) The higher $v$ of the region related to the absorption is, the lower the peak temperature of the absorption is. (2) When the number of $\mathrm{OH}$ groups in a solvent molecule is the same, $v$ related to $\alpha_{22}, \alpha_{21}$, and $\alpha_{12}$ absorptions decreases with increase in molecular weight. (3) When molecular weight is the same, $v$ related to $\alpha_{22}, \alpha_{21}$, and $\alpha_{12}$ absorptions for the solvent with two $\mathrm{OH}$ groups in one molecule is larger than that of the solvent with one $\mathrm{OH}$ group. (4) As for $\alpha_{\mathrm{sh}}$ absorption, $v$ in $n$-alcohols showed an asymptotic value of $c a$. 0.35. (5) When we compare the values of $v$ for NP and BMM40, BMM40 is always larger than NP. This indicates $v$ is partly governed by the diffusion of the solvent into the primary particles. Here, the accuracy of $v$ evaluated through the relative error was $c a .10 \%$ of the absolute value of $v$.

\section{REFERENCES}

1. S. Manabe, Kobunshi, 32, 84 (1983).

2. S. Manabe, "Diffusion in Polymer," P. Neogi, Ed., Marcel Dekker, New York, N.Y. 1996.

3. M. Mitsuishi, Y. Naruoka, M. Shimizu, K. Hamada, and T. Ishiwata, J.S.D.C., 112, 333 (1996).

4. M. L. Gulrajani and R. M. Saxena, J.S.D.C., 95, 330 (1979)

5. H. Fujita, Fortschr. Hoch. Polymer Forsch., 3, 1 (1961).

6. S. Manabe, M. Iwata, and K. Kamide, Polym. J., 18, 1 (1986).

7. S. Manabe and R. Fujioka, Polym. J., 20, 860 (1996).

8. for example, J. D. Ferry, "Viscoelastic Properties of Polymers," John Wiley \& Sons, New York, N.Y., 1961 and 1970.

9. for example, A. E. Woodward, J. A. Sauer, and R. A. Wall, J. Chem. Phys., 30, 854 (1959).

10. S. Manabe, S. Uemura, and M. Takayanagi, Kogyo Kagaku Zasshi, 70, 529 (1967).

11. J. M. Kure, A. P. Pierlot, and I. M. Russel, Textile Res. J., 67, 18 (1997).

12. G. P. Johari, A. Hollbrucker, and E. Mayer, Nature, 330, 552 (1987).

13. J. S. Vrentas and C. M. Vrentas, Macromolecules, 27, 4684 (1994).

14. S. Uemura and M. Takayanagi, J. Appl. Polymer Sci., 10, 113 (1966). 